\title{
A Quantitative Theory of Information and Unsecured
}

\section{Credit: Additional Appendix}

\author{
Kartik Athreya* \\ Xuan S. Tam ${ }^{\dagger}$ \\ Federal Reserve Bank of Richmond University of Cambridge \\ Eric R. Young \\ University of Virginia
}

January 3, 2012

\section{Appendix: Not For Publication}

\subsection{Computing Partial Information Equilibria}

The imposition of conditions on beliefs off-the-equilibrium path makes the computational algorithm we employ relevant for outcomes, so we discuss in some detail our algorithm for computing partial information competitive equilibria. The computation of the full information equilibrium is straightforward using backward induction; since the default probabilities

\footnotetext{
* Corresponding author. Research Department, Federal Reserve Bank of Richmond, kartik.athreya@rich.frb.org.

$\dagger$ Centre for Financial Analysis \& Policy, Judge Business School, University of Cambridge, xst20@cam.ac.uk.

$¥$ Department of Economics, University of Virginia, ey2d@virginia.edu.
} 
are determined by the value function in the next period, we can solve for the entire equilibrium, including pricing functions, with one pass. The partial information equilibrium is not as simple, since the lender beliefs regarding the state of borrowers influence decisions and are in turn determined by them; an iterative approach is therefore needed.

1. Fix an agent type by observables $(y, j, m, \chi)$

2. Guess the initial function $q^{(0)}\left(b^{\prime}, y, j, m, \chi\right)$ discussed above

3. Solve household problem to obtain $b^{\prime}=g(b, y, e, \nu, \chi, \lambda, j, m), S_{\{e, \lambda\}}$, and $d\left(b^{\prime}, e^{\prime}, \nu^{\prime}, \chi^{\prime}, \lambda^{\prime}\right)$, and $\Gamma(\cdot)$.

4. For all $b^{\prime}$ observed, use $S_{\{e, \lambda\}}$ and $\Gamma(\cdot)$ to compute $\operatorname{Pr}\left(e, \lambda \mid b^{\prime}, y, j, m, \chi\right)$ the probability that an individual is in $(e, \lambda)$ given observed $\left(b^{\prime}, y, j, m, \chi\right)$. Knowledge of $\operatorname{Pr}\left(e, \lambda \mid b^{\prime}, y, j, m, \chi\right)$ and the distribution of households over the remaining observable state variables implies $\Upsilon\left(\Omega \mid b^{\prime}\right)$.

5. Compute

$\widehat{\pi}^{b}\left(b^{\prime}, y, j, m, \chi\right)=\sum_{e} \sum_{\nu} \sum_{\lambda} \sum_{\chi^{\prime}} \pi_{\chi}\left(\chi^{\prime}\right) \pi^{b^{\prime}}\left(b^{\prime}, y, e, \nu, \chi, \lambda, j, m\right) \operatorname{Pr}\left(e, \lambda \mid b^{\prime}, y, j, m, \chi\right)$,

the expected probability of default for an individual in observed state $\left(b^{\prime}, y, j, m, \chi\right)$;

6. Fill in the "holes" in $\widehat{\pi}^{b^{\prime}}$ for all $b^{\prime}$ not observed by applying the interim off-equilibrium beliefs as described in the main text.

7. Compute an "intermediate" price function $\widehat{q}$ for all $b^{\prime}$, that is actuarially fair (competitive) given the preceding estimate $\widehat{\pi}^{b^{\prime}}$ :

$$
\widehat{q}\left(b^{\prime}, y, j, m, \chi\right)=\frac{\left(1-\widehat{\pi}^{b^{\prime}}\left(b^{\prime}, y, j, m, \chi\right)\right) \psi_{j}}{1+r+\phi} \quad \text { for all } b \geq b_{\min }(b, y, j, m, \chi)
$$


8. Set

$$
q^{(1)}\left(b^{\prime}, y, j, m, \chi\right)=\Xi q^{(0)}\left(b^{\prime}, y, j, m, \chi\right)+(1-\Xi) \widehat{q}\left(b^{\prime}, y, j, m, \chi\right)
$$

where $\Xi$ is set very close to 1 (we use 0.985 ), return to Step 1 and repeat until the pricing function converges.

Given a pricing function, households make decisions about debt. For all debt levels selected by positive fraction of households, we can then compute the likelihood of default as a function of their observable type. These are Steps 1-5 in the algorithm. Next, for debt levels that lie in any range not chosen by any households, we proceed as follows. Let $k$ be the index of iteration, let $q^{(k)}$ and be the current price function, and $\mu^{(k)}$ be the associated distribution of debt requests induced by agent decisions, given $q^{(k)}$. For any given debt request $b^{\prime}$ such that $\mu^{(k)}\left(b^{\prime}\right)=0$, define $b^{\prime}<b^{-}<0$ as the nearest lower debt level relative to $b^{\prime}$ at which $\mu^{(k)}\left(b^{\prime}\right)>0 . b^{-}$is therefore the upper end (or right-endpoint) of a segment of debt levels no household requests, given $q^{(k)}$. Define $\widehat{q}\left(b^{-}, I\right)$ to be the actuarially fair price at $b^{-}$; this is where we use optimal inference by lenders to construct $\widehat{\pi}^{b^{\prime}}$.

Next, define $b^{+}<b^{\prime}<0$ to be the nearest higher debt level (i.e. a more negative value for assets) relative to $b^{\prime}$, at which $\mu^{(k)}\left(b^{+}\right)>0 . b^{+}$is therefore the lower end (or left-endpoint) of the same segment of debt levels, again given $q^{(k)}$. Denote by $\widehat{q}\left(b^{+}, I\right)$ the actuarially fair price at $b^{+}$. Thus, at any $b, \widehat{q}(b, I)$ is the actuarially fair price at any debt level that is observed under $\mu^{(k)}$, and equal to $\widehat{q}\left(b^{-}, I\right) \forall b \in\left(b^{+}, b^{-}\right]$. The collection of these segments is then denoted $\widehat{q}(\cdot, I) .{ }^{1}$ With $\widehat{q}$ in hand, as well as the current iterate for the pricing function $q^{(k)}$, we construct the next iterate of the pricing function, $q^{(k+1)}$ as a convex combination of $q^{(k)}$ and $\widehat{q}(\cdot, I)$ that places weight $\Xi$ on $q^{(k)}$. The preceding is Step 6 .

We want to point our here that we do not confront the agent with the function $\widehat{q}$; we cannot guarantee that the sequence of $\widehat{q}$ functions is monotone. Instead, we update the

\footnotetext{
${ }^{1}$ Note that $\widehat{q}$ is a (lower semicontinuous) step-function.
} 
pricing functions extremely slowly using a weight $\Xi$ on the current iterate $q^{(k)}$ close to one (we use $\Xi=0.985$ ), and thereby obtain a monotone sequence of pricing functions (see the proof of convergence below). This procedure constitutes Steps 7 and 8 in the algorithm. Repeating this procedure to convergence, we obtain the equilibrium pricing function and loan request distribution. In addition, note that the segments starting at $b^{-}$will, in subsequent iterations, lead those households at $b^{-}$to potentially lower their borrowing slightly in the next iteration, as they receive discontinuously better pricing for doing so, even under the heavily convexified pricing function we will face them with at that point. ${ }^{2}$ As a result, some pooling can occur in equilibrium as agents of different types are inframarginal at any discrete jumps in loan pricing. We discuss measures of pooling in the main text extensively.

Because the household value function is continuous but not differentiable or concave, we solve the household problem on a finite grid for $b^{\prime}$, using linear interpolation to evaluate the value function at points off the grid. Similarly, we use linear interpolation to evaluate $q$ at points off the grid for $b^{\prime}$. To compute the optimal savings behavior we use golden section search (see Press et al. 1993 for details of the golden section algorithm) after bracketing with a coarse grid search; we occasionally adjust the brackets of the golden section search to avoid the local maximum generated by the nonconcave region of the value function. To calibrate the model we use a derivative-free method to minimize the sum of squared deviations from the targets; the entire program is implemented using OpenMPI instructions in Fortran95 on a 32-processor Mac cluster. We make no claims about the program working for parameter values we have not explored. The computational cost of the PI equilibria is very large (roughly ten days of computing time per equilibrium), and our attempts to speed it up have led to equilibria with substantially less borrowing.

We next provide a proof that the numerical procedure has a maximal fixed point, and that this fixed point is the one we obtain. Because the machinery used for this proof may

\footnotetext{
${ }^{2}$ We thank a referee for bringing this to our attention.
} 
not familiar to all readers, we provide some basic definitions as well.

\subsection{Proof of Convergence}

Define $Q$ to be a collection of nondecreasing step functions defined over a finite set of points $\mathcal{D} \subset \mathcal{R}$; the number of steps is therefore necessarily finite, so $Q$ itself is finite. Let $Q$ contain a maximal element $\mathbf{1}$ and a minimal element $\mathbf{0}$, using the pointwise ordering of functions ( $f \precsim g$ if and only if $f(b) \leq g(b) \forall b \in \mathcal{D})$; suppose all such members can be ordered. Endow $Q$ with the Scott topology, which defines order-continuity: a function $\Phi: Q \rightarrow Q$ is order-continuous (or Scott-continuous) if $\sup \{\Phi(R)\}=\Phi\{\sup (R)\}$ for each $R \subset Q$. Since $Q$ is finite, order-continuity reduces to pointwise convergence in $\mathcal{R}^{n}$.

Define $\Phi: Q \rightarrow Q$ as the mapping defined in the algorithm; heuristically, $\Phi$ takes a given loan pricing function $q^{0}$, constructs the break-even pricing function $\widehat{q}$ implied by behavior of agents confronted with $q^{0}$, and then produces a new pricing function $q^{1}$ as a convex combination of the two functions:

$$
\Phi\left(q^{0}\right)=\Xi \widehat{q}\left(q^{0}\right)+(1-\Xi) q^{0}
$$

for some $\Xi \in[0,1]$. Define $\Phi^{n}\left(q^{0}\right)$ as $\Phi$ composed $n$ times.

Lemma $1.1(Q, \precsim)$ is a complete lattice.

Proof $(Q, \precsim)$ is clearly a lattice, as it is a collection of lower-semicontinuous step functions that are nonincreasing and ordered pointwise. To see that it is a complete lattice, note that an arbitrary collection of lower-semicontinuous functions has a pointwise supremum, and $\mathbf{0}$ bounds all collections from below. Therefore, following Davey and Priestley (2002) (Q, $)$ is a complete lattice.

We will apply the following theorem (see Granas and Dugundji 1986). 
Theorem 1.2 (Tarski-Kantorovitch) Let $(Q, \precsim)$ be a partially-ordered set and $\Phi: Q \rightarrow Q$ be order-continuous. Assume there exists $q \in Q$ such that (i) $q \geq \Phi(q)$ and (ii) every countable chain in $\{x \mid x \leq q\}$ has an infimum. Then the set of fixed points of $\Phi$ is not empty. Furthermore, $q^{*}=\inf _{n}\left\{\Phi^{n}(q)\right\}$ is a fixed point and $q^{*}$ is the maximum of the set of fixed points of $\Phi$ in $\{x \mid x \leq q\}$.

Any complete lattice is a partially-ordered set.

Let $d=1$ denote the decision to default next period, and $\operatorname{Pr}(d=1 \mid b)$ denote the probability of default next period conditional on issuing debt $b$ today.

Proposition 1.3 $\operatorname{Pr}(d=1 \mid b)$ is nondecreasing given $q(b)$. That is, the probability of default weakly rises with the amount of debt, holding fixed pricing.

Proof See Chatterjee et al. (2007), Theorem 6.

Conjecture 1.4 $\operatorname{Pr}(d=1 \mid q)$ is nonincreasing at each b. That is, the probability of default on a given amount of debt is weakly falling in the price of that debt.

This conjecture rules out the possibility that two iterates $q^{n}$ and $q^{n+1}$ "cross" each other, in that $q^{n}(b)>q^{n+1}(b)$ for some $b$ and $q^{n}(b)<q^{n+1}(b)$ for other $b$; that is, it implies that $\left\{\widehat{q}\left(q^{n}\right)\right\}$ is a monotone chain. The economic content of the conjecture is that the pool of borrowers who choose a particular $b$ level in equilibrium cannot improve as $q$ falls, implying that the equilibrium default rate on that debt level must weakly rise. The conjecture does not appear to be provable in general, but it is satisfied by the numerical procedure we use. Fortunately, the proof of convergence below does not require $\left\{\widehat{q}\left(q^{n}\right)\right\}$ to be a monotone chain, only $\left\{q^{n}\right\}$; with careful choice of $\Xi$ we have been able to guarantee monotonicity of $\left\{q^{n}\right\}$ in all cases we examined.

Theorem 1.5 $\Phi$ has a maximal fixed point $q^{*}=\Phi\left(q^{*}\right)$. Furthermore, $\left\{\Phi^{n}(\mathbf{1})\right\} \rightarrow q^{*}$. 
Proof Under Conjecture 1.4, $\Phi$ is a monotone nonincreasing mapping in the pointwise ordering $\left(q^{n} \succeq q^{n+1}\right) . \Phi$ is order-continuous because the sequence $\left\{q^{n}(b)\right\}$ is monotone for each $b$ and confined to a compact set $[0,1]$. By the Tarski-Kantorovitch theorem, the set

of fixed points is nonempty and the chain $\left\{\Phi^{n}(\mathbf{1})\right\} \rightarrow q^{*}$, the maximal element of the set of fixed points.

Uniqueness is not generally assured, since $q=0$ is a fixed point; uniqueness therefore only obtains when there does not exist any fixed point with $q \geq 0$. A sufficient condition for $q^{*} \neq 0$ is that $\Lambda>0$; in that case, no default will occur at $b>-\Lambda$ so $q=0$ is never the maximal fixed point. A separate sufficient condition is $\lambda>0$, since again there will exist a small enough debt level that will never be defaulted on, although it is not possible to characterize analytically where this debt level lies. Necessary conditions for $q^{*} \neq 0$ are unknown.

Any equilibrium $q$ must be a fixed point of $\Phi$. Since our program converges to the maximal fixed point, it converges to the competitive equilibrium with the lowest interest rate functions. This equilibrium has the property that, with an exogenous risk-free rate $r$, budget sets are largest under full information (and therefore consumer welfare is maximized). Under asymmetric information the first statement still holds (budget sets are larger when interest rates are lower), but utility may not be maximized for all individuals due to the potential for pooling; nevertheless, we think that this equilibrium is the natural one to study.

\subsection{The Roles of $\lambda$ and $\Delta$ Under Full Information}

The non-pecuniary cost of bankruptcy, $\lambda$, plays an important role in allocations. It is meant to capture various aspects of deadweight costs borne by households in bankruptcy. There are two key aspects to this process - the coefficient of variation and the persistence. If 
one forces $\lambda$ to remain constant and uniform across households when it is chosen to match the observed filing rate, the model produces a too-small discharge-income ratio and can no longer capture the heterogeneity in default costs implied by the estimates of Fay, Hurst, and White (1998). The calibrated value of $\lambda$ in this case is also too small, in the sense that the model generates counterfactually-small bankruptcies, and as a result will understate the welfare costs of frequent default. If $\lambda$ differs across households but is iid, discharge rates remain too low as the average $\lambda$ needs to be small. Without persistence, no household's implicit collateral is expected to be particularly valuable in the next period and thus cannot support large debts. Thus, our calibration allows for the high cross-sectional dispersion and high persistence in $\lambda$ needed in order to jointly support (i) large risky debts on which default premia are paid, (ii) frequent default, and (iii) relatively large discharges. If we change the average $\lambda$ in the economy, the effect is to move default rates and discharge levels in opposite directions (see Athreya 2004). Furthermore, changing merely the average $\lambda$ delivers little change in the dispersion in the terms. Thus, changes in stigma can be dismissed as the force driving all of the changes in the unsecured credit market.

Lastly, we discuss the roles played by the two main transactions costs, $\Delta$ and $\phi$. As noted in Livshits, MacGee, and Tertilt (2007) and Athreya (2004), dropping transactions costs can potentially deliver the trends in the default rates and debts observed in the data, so these changes are worth examining as competitor stories. No household in the model would default on any debt less than this cost (i.e, when $b>-\Delta$ ), so higher values of $\Delta$ can support larger debts in general. Changes in $\Delta$ only alter the length of the initial flat segment where risk-free borrowing is sustained (Figure 1 plots price $q$ as a function of borrowing $b$ ). Changes in $\phi$ only shift the pricing functions up and down (see Figure 1), altering the cost of issuing any given amount of debt. Thus, neither change will alter the variance of interest rates that agents receive, as they affect all agents symmetrically. To get a change in the distribution of interest rates, one needs to generate changes in the slope of the pricing functions. As a result, 
stories that place falling transactions costs at the heart of the changes in the unsecured credit market cannot account for the homogeneity observed in the earlier period. More details on experiments with $(\lambda, \Delta, \phi)$ are available upon request.

Having given a flavor of how pricing works in the model, we turn now to credit availability under full information; the 'supply side' of the credit market is seen most clearly in the pricing of debt facing households in varying states of income. For questions regarding unsecured credit, the young are the most relevant population, and we therefore focus on their access to credit. Figure 1 in the main text displays the pricing functions for college types at age 29 given both the low and high value of $\lambda$; as would be expected the higher the realization of $e$ the more credit is available (at any given interest rate). For low realizations of $e$ the pricing functions look like credit lines - borrowing can occur at a fixed rate (in this case, the risk-free rate) up to some specified level of debt, after which the interest rate goes to $\infty$. For higher realizations the increase in the interest rate is more gradual, meaning that some risky borrowing will occur in equilibrium; for some borrowers, the marginal gain from issuing debt is sufficiently high that they are willing to pay a default premium to do it. The pricing functions for noncollege types look similar but involve higher interest rates at any given level of debt. Similar pictures arise for older agents - they are weakly decreasing in debt with more gradual increases in interest rates for luckier agents. Middle-aged agents (say, age 45) can borrow significantly more than their younger counterparts, although they choose not to do so in equilibrium because they are saving for retirement. Given a high value of $\lambda$ agents can borrow a lot more (as would be expected).

Lastly, a reason for our focus on improved information as a candidate explanation for the facts is the increase in dispersion of credit terms observed (and so paid in equilibrium). Figure 3 displays model's implications for the evolution of the variance of equilibrium borrowing rates over the life-cycle across the two main information regimes we consider. These rates are not weighted by the amount of debt, they are direct measures of dispersion computed 
identically to what we measure from the SCF. The dispersion in interest rates is fairly flat over the life-cycle, and the variance is systematically higher for the less educated groups, since those groups are the ones who borrow and default on the equilibrium path. In the model, agents are willing to pay fair premiums for the option to default and do so. Furthermore, since all pricing is actuarially-fair with respect to default risk, agents who pose higher risk will pay higher prices to borrow. We also note that in general, the less well educated pay higher interest rates throughout life than their better educated counterparts. Given the earnings process, this is not surprising. However, what is interesting here is that the model suggests that partial information may lead to lower average interest rates, or alternatively, that improvements in information may well coincide with the observation of more households borrowing at higher interest rates. The reason is intuitive, and reflects the pricing functions displayed earlier. In essence, loan interest rates under partial information are frequently high enough to discourage borrowing.

\subsubsection{Information and the "Causes" of Bankruptcy}

Here, we detail the joint role played by expenditure shocks and non-pecuniary costs of bankruptcy. The results are given in Table 1. Each cell in which the information regime is held fixed presents the joint distribution of expenditure shocks and non-pecuniary costs of those who have filed for bankruptcy. What is clear is that under all information regimes the bulk of filers have low non-pecuniary costs (high $\lambda$ ). More interestingly, defaulting households typically have not received the largest expenditure shock. Specifically, the median

expenditure shock is zero, and yet households with this realization account for more than half of all filers. Perhaps the most interesting finding here is that as that information becomes more limited, we see that the fraction of households in bankruptcy who have a low nonpecuniary cost of filing and have received no expenditure shock grow dramatically. This result suggests that "strategic bankruptcy" is certainly a real possibility under PI regimes 
in a way that is more severely restricted under FI. In particular, while the latter group accounts for 46 percent under FI, they are 76 percent of all filers under $\operatorname{PI}(\lambda, e)$. With respect to income, Figure 4 shows that most defaulting households have not experienced extremely bad transitory shocks.

Despite the possibility that the joint distribution of expenditure shocks and non-pecuniary costs suggests some strategic filing, what is still true is that the high expenditure shock is rare, and so may not be seen often among filers for that reason alone. Nonetheless, such a shock may well "push" someone into bankruptcy when it occurs. Table 2 presents the conditional probability of bankruptcy, given a particular constellation of the two variables of interest. It is clear here that the high shock does indeed "cause" a disproportionate amount of bankruptcy, relative to its unconditional likelihood. Moreover, Table 2 makes clear the power of this shock: conditional on getting this bad expenditure shock, the probability of bankruptcy is not highly sensitive to either the non-pecuniary cost or the information regime that prevails. By contrast, filing probabilities vary much more substantially across information and non-pecuniary costs when the expenditure shocks are smaller.

\subsection{Tables}

Table 1: Expenditure Shocks and Non-Pecuniary Costs Among Filers

\begin{tabular}{|l|cc|cc|cc|cc|}
\hline \hline & \multicolumn{2}{|c|}{ FI } & \multicolumn{2}{c|}{ PI $(e)$} & \multicolumn{2}{c|}{ PI $(\lambda)$} & \multicolumn{2}{c|}{ PI $(\lambda, e)$} \\
\hline & High $\lambda$ & Low $\lambda$ & High $\lambda$ & Low $\lambda$ & High $\lambda$ & Low $\lambda$ & High $\lambda$ & Low $\lambda$ \\
\hline Low $\chi$ & 0.3776 & 0.0118 & 0.1664 & 0.0065 & 0.0405 & 0.0000 & 0.0496 & 0.0000 \\
Median $\chi$ & 0.4621 & 0.0623 & 0.6299 & 0.0755 & 0.7793 & 0.0393 & 0.7608 & 0.0476 \\
High $\chi$ & 0.0515 & 0.0346 & 0.0725 & 0.0492 & 0.0849 & 0.0561 & 0.0853 & 0.0567 \\
\hline
\end{tabular}


Table 2: Expenditure Shocks and Non-Pecuniary Costs as Triggers

\begin{tabular}{|l|rr|rr|rr|rr|}
\hline \hline & \multicolumn{2}{|c|}{ FI } & \multicolumn{2}{c|}{ PI $(e)$} & \multicolumn{2}{c|}{ PI $(\lambda)$} & \multicolumn{2}{c|}{ PI $(\lambda, e)$} \\
\hline & High $\lambda$ & Low $\lambda$ & High $\lambda$ & Low $\lambda$ & High $\lambda$ & Low $\lambda$ & High $\lambda$ & Low $\lambda$ \\
\hline Low $\chi$ & $0.964 \%$ & $0.030 \%$ & $0.385 \%$ & $0.015 \%$ & $0.068 \%$ & $0.000 \%$ & $0.077 \%$ & $0.000 \%$ \\
Median $\chi$ & $15.380 \%$ & $2.071 \%$ & $18.986 \%$ & $2.276 \%$ & $17.123 \%$ & $0.863 \%$ & $15.430 \%$ & $0.965 \%$ \\
High $\chi$ & $26.422 \%$ & $17.751 \%$ & $33.728 \%$ & $22.889 \%$ & $28.792 \%$ & $19.025 \%$ & $26.702 \%$ & $17.750 \%$ \\
\hline
\end{tabular}

Table 3: Credit Sensitivity

\begin{tabular}{|l|rr|rr|}
\hline \hline & \multicolumn{2}{|c|}{$b<0, m=0$} & \multicolumn{2}{c|}{$b<0, m=1$} \\
\hline Mean & $b$ & $r$ & $b$ & $r$ \\
\hline \hline COLL & 0.0718 & $7.33 \%$ & 0.0688 & $9.74 \%$ \\
HS & 0.0402 & $9.58 \%$ & 0.0341 & $12.55 \%$ \\
NHS & 0.0310 & $12.16 \%$ & 0.0285 & $13.71 \%$ \\
\hline
\end{tabular}

\section{$1.5 \quad$ Figures}

\section{References}

[1] Davey, Brian A. and Hilary A. Priestley. 2002. Introduction to Lattices and Order, Second Edition. Cambridge Press.

[2] Granas, Andrzej and James Dugundji. 1986. Fixed Point Theory. Springer Publishing.

[3] Press, William H., Saul A. Teukolsky, William T. Vetterling, and Brian P. Flannery. 1993. Numerical Recipes in FORTRAN: The Art of Scientific Computing. Cambridge University Press. 
Figure 1: The Role of Transactions Costs

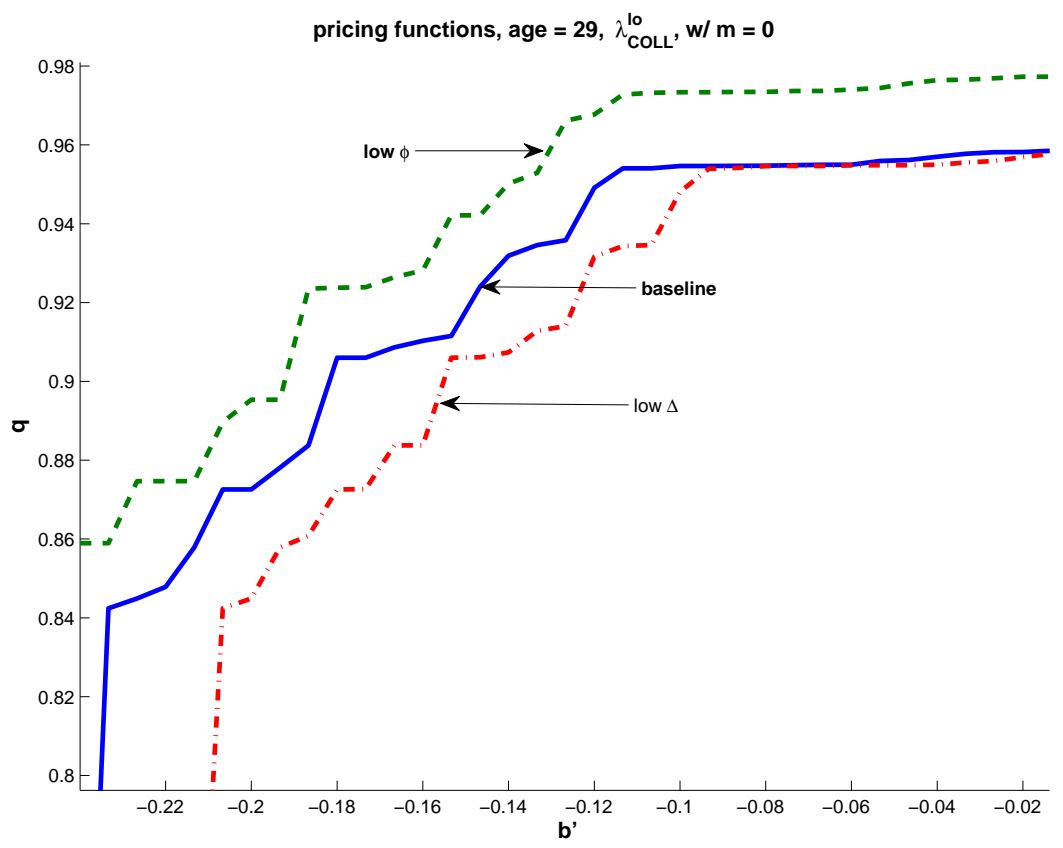

Figure 2: Labor Productivity over the Life-cycle

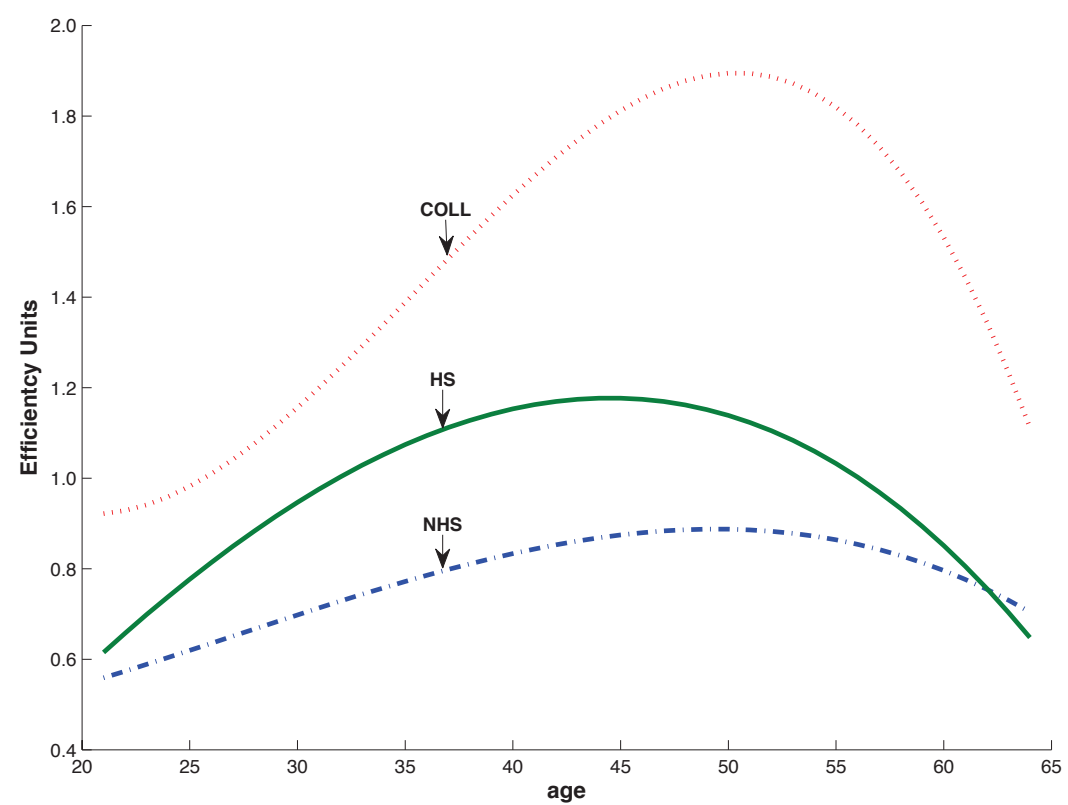


Figure 3: Variance of Equilibrium Borrowing Rates over the Life-Cycle

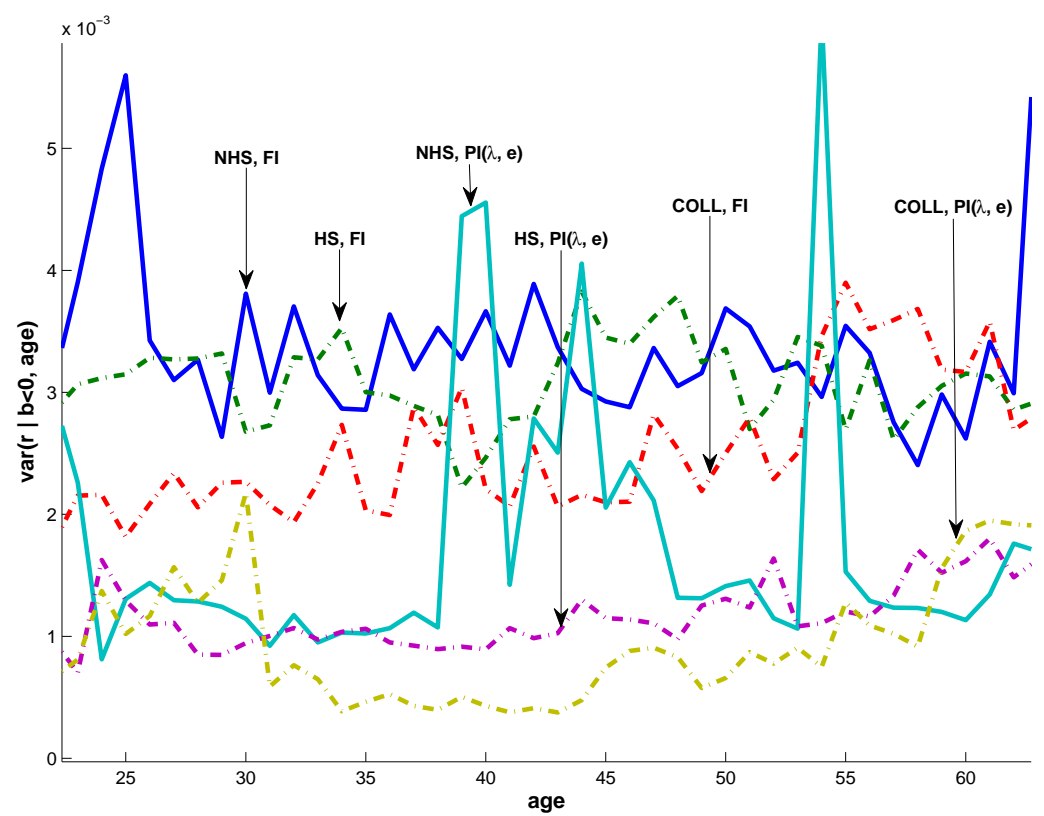

Figure 4: The Income Shocks of Defaulters

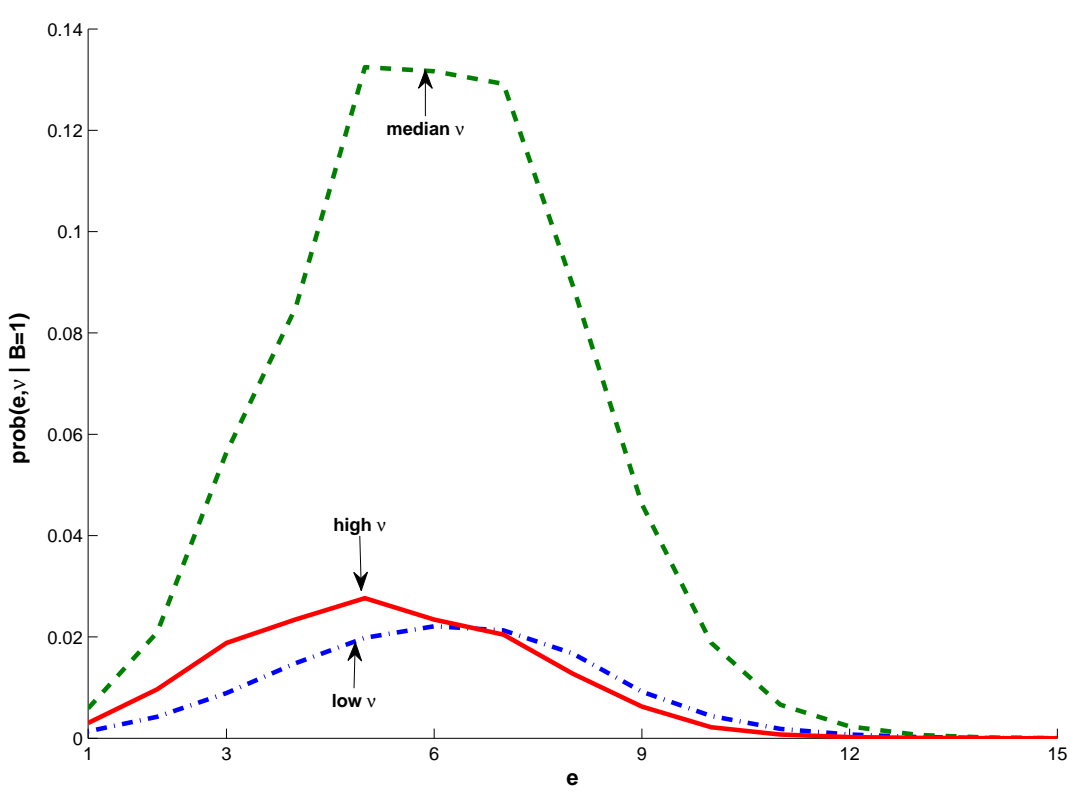


Figure 5: Credit Supply Under Full Information

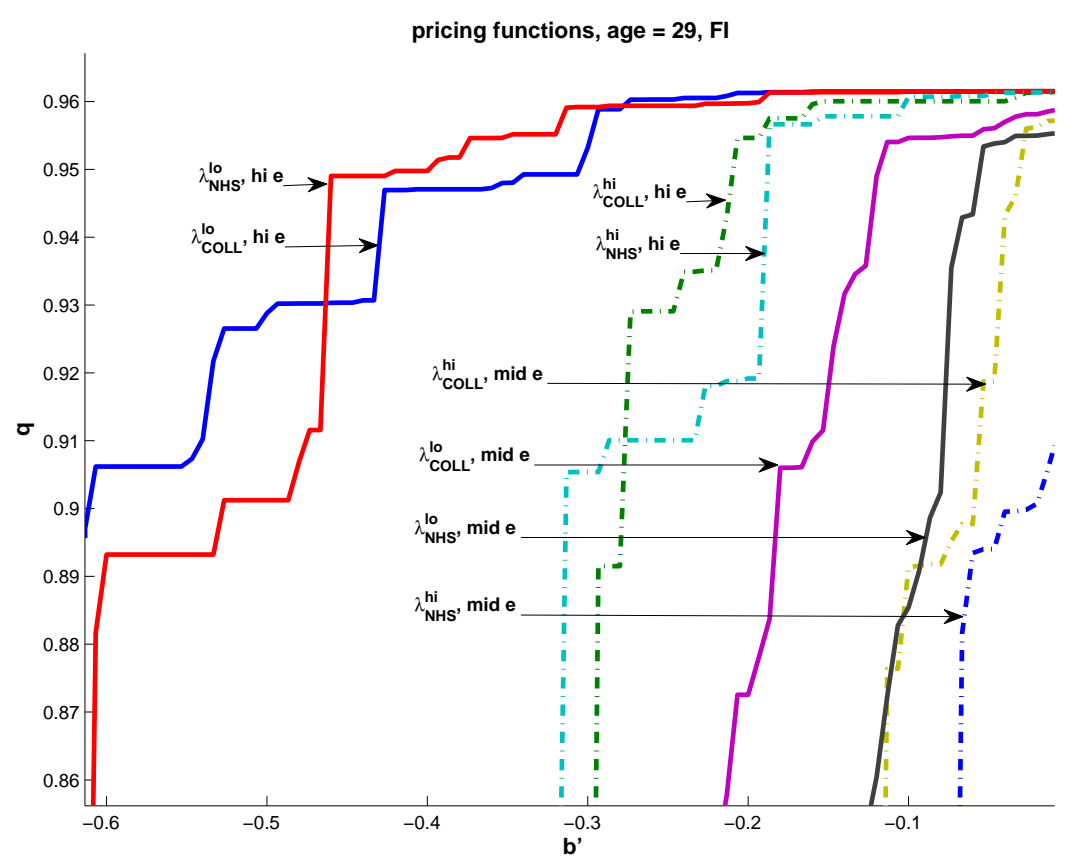

